\title{
Caregiver Burden of Rheumatoid Arthritis Patients With Self-Care Deficit in China: A Cross-Sectional Study
}

\author{
Guoxia HE, Yu DING
}

Department of Rheumatology, The First Affiliated Hospital of Nanjing Medical University, Nanjing, China

\begin{abstract}
Objectives: This study aims to investigate the level of caregiver burden of Chinese rheumatoid arthritis (RA) patients with self-care deficit. Patients and methods: This cross-sectional study included a total of 65 caregivers (30 males, 35 females; mean age 52 years; range 20 to 79 years) of 65 RA inpatients ( 9 males, 56 females; mean age 59 years; range 20 to 85 years) with self-care deficit. Demographic data of patients and their respective caregivers were collected. The level of caregiver burden was defined using Caregiver Burden Inventory, a 24-item-five-domain survey tool. Correlation between different demographic factors and caregiver burden was analyzed. Predictive factors for burden level were also investigated.

Results: A mean Caregiver Burden Inventory score of $44.0 \pm 4.0$ was observed among caregivers of Chinese RA patients with self-care deficit. Among the five Caregiver Burden Inventory domains, developmental burden scored the highest (11.7 \pm 1.4$)$, while physical burden scored the lowest $(6.7 \pm 1.0)$. Among various demographic factors, patients' age $(r=0.306, p=0.013)$ and health insurance coverage $(r=-0.246, p=0.04)$ were correlated with the level of caregiver burden. Besides, caregiver's educational level $(r=-0.316, p=0.01)$, relationship of caregiver with the patient $(r=0.355, p=0.004)$, and whether or not the caregiver lived with the patient $(r=0.362, p=0.003)$ were also significant factors. Predictive factors for caregiver burden of RA patients were identified as patient's health insurance coverage and caregiver's relationship with the patient, in agreement with the correlation analysis.

Conclusion: Since caregiver burden may contribute to adverse health outcomes, supportive interventions should be established to target Chinese RA patients with self-care deficit and their caregivers to consolidate the sustainable care provided both at hospital and home.

Keywords: Burden; caregiver; rheumatoid arthritis; self-care deficit.
\end{abstract}

Rheumatoid arthritis (RA) is a common autoimmune disorder characterized by chronic inflammation, which primarily involves joints. ${ }^{1}$ In advanced stages of RA, deformity of joints and consequent dysfunction may severely affect patients' quality of life and result in self-care deficit. Due to the chronicity of RA, caring for these patients may be associated with enduring stress and compromised psychosocial functioning of caregivers. Since both physically and mentally healthy caregivers are critical to effective and sustainable caring for RA patients, it is of significance to evaluate the areas of greatest distress and their relevant factors. Such an insight may also assist in formulating a strategy to alleviate caregiver stress and ultimately improve the quality of life in RA patients. Ever since the 1960s, "caregiver burden" has been postulated to refer to negative experiences of caregivers, both subjective and objective, and study of caregiver burden has thus become an important arena of modern care research. ${ }^{2}$ In China, the burden of caring for RA patients is largely born by informal caregivers and data concerning their stress are lacking. Therefore, in this study, we aimed to investigate the level of caregiver burden of Chinese RA patients with self-care deficit. 


\section{PATIENTS AND METHODS}

The study was conducted between August 2012 and October 2014 and approved by the Ethics committee of the First Affiliated Hospital of Nanjing Medical University. The study included a total of 65 caregivers (30 males, 35 females; mean age 52 years; range 20 to 79 years) of 65 Chinese RA inpatients (9 males, 56 females; mean age 59 years; range 20 to 85 years) with self-care deficit. The inclusion criteria for RA patients were as follows; confirmed diagnosis as RA according to the 2010 criteria proposed by American College of Rheumatology/European League Against Rheumatism, ${ }^{3}$ a disease duration $\geq 5$ years, Health Assessment QuestionnaireDisability Index $\geq 1.5$, Disease Activity Score 28 $>5.1$, and Global Functional Status of class IV. Exclusion criteria included those who underwent joint replacement surgery or biologic therapy, or those having severe cardiovascular comorbidity or mental problems. Informal caregiver was defined as a family member of the patient who was primarily responsible for constant and daily caring for at least three months. Caregivers who were financially compensated, mentally unstable or difficult to communicate were also excluded. A written informed consent was obtained from each patient. The study was conducted in accordance with the principles of the Declaration of Helsinki.

Basic demographic data of RA patients including age, sex, educational level, and financial sufficiency were collected, while data such as age, sex, marital status, occupation, educational level, relation to the patient, self-perceived health status, and time of caring per day were included for caregivers. Caregiver stress was evaluated using Caregiver Burden Inventory (CBI), ${ }^{4}$ the Chinese version of which has previously been validated. ${ }^{5}$ $\mathrm{CBI}$ is a multidimensional measure of caregiver burden, consisting 24 items/questions in five domains: time-dependent burden, developmental burden, social burden, physical burden, and emotional burden. The questions are designed to assess different aspects of physical, mental as well as social stress associated with long-term caring, each of which is rated on a 0 to 4-point scale in terms of the frequency of experiencing the stress: 0 , never; 1 , rarely; 2 , sometimes; 3 , quite frequently; 4 , nearly always. The total score ranges from 0 to 96 , growing in proportion to the overall severity of the stress perceived by the caregiver. ${ }^{3}$

\section{Statistical analysis}

Caregivers enrolled in the study were requested to complete the questionnaire independently. For those who had difficulties in reading or understanding the items, the researchers provided necessary assistance without influencing their judgment. Statistical analysis was performed using SPSS version 16.0 (SPSS Inc., Chicago, IL, USA). Independent samples $\mathrm{T}$ test and one-way analysis of variance test were used to assess the caregiver burden scores among different demographic groups. Spearman's rank correlation coefficient test was performed to assess the direction and magnitude between demographic variables and the caregiver burden. Possible predictive factors for caregiver burden was further determined via stepwise multivariate regression analysis. The level of significance was defined as $p<0.05$.

\section{RESULTS}

Caregiver burden levels of 65 RA patients and their primary caregivers were summarized in Table 1. Mean score of CBI for the caregivers was $44.0 \pm 4.0$ (range, 35 to 52). Among five domains, developmental burden and time-dependent burden scored the highest $(11.7 \pm 1.4$ and $10.1 \pm 1.6$, respectively), followed by social burden $(8.6 \pm 1.2)$. Emotional burden $(6.9 \pm 1.4)$ and physical burden $(6.7 \pm 1.0)$ had the lowest scores. Among 24 items, eight items (five of developmental burden and three of time-dependent burden) demonstrated a mean score of more than two points: (i) I expected that things would be different at this point in my life (2.6 0.6), (ii) I wish I could escape from this situation (2.4 0.6), (iii) I feel that I am missing out on life (2.3 0.6), (iv) My social life has suffered (2.3 0.6), (v) I feel emotionally drained due to caring for him/her (2.2 0.6), (vi) I do not have a minute's break from his/her chores (2.2 0.6), (vii) $\mathrm{He} /$ she needs my help to perform many daily tasks (2.1 0.7), and (viii) I have to help him/her with many basic functions (2.1 0.6).

Demographic characteristics of RA patients with self-care deficit and their caregivers 


\begin{tabular}{|c|c|c|c|c|}
\hline \multirow[t]{2}{*}{ Demographic variables } & \multirow[t]{2}{*}{ No. of caregivers } & \multirow{2}{*}{$\frac{\mathrm{CBI} \text { score }}{\text { Mean } \pm \mathrm{SD}}$} & \multirow{2}{*}{$\begin{array}{l}t / F \\
(p)\end{array}$} & \multirow{2}{*}{$\begin{array}{l}\text { Rho } \\
(p)\end{array}$} \\
\hline & & & & \\
\hline \multicolumn{5}{|l|}{ Patients } \\
\hline \multicolumn{5}{|l|}{ Age (years) } \\
\hline$>50$ & 46 & $44.8 \pm 3.6$ & -2.526 & 0.306 \\
\hline$\leq 50$ & 19 & $42.1 \pm 4.4$ & $(0.014 *)$ & (0.013*) \\
\hline \multicolumn{5}{|l|}{ Sex } \\
\hline Male & 9 & $43.7 \pm 5.2$ & -0.254 & 0.014 \\
\hline Female & 56 & $44.0 \pm 3.9$ & $(0.800)$ & $(0.910)$ \\
\hline \multicolumn{5}{|l|}{ Educational level (years) } \\
\hline$>9$ & 10 & $43.10 \pm 4.01$ & -0.755 & 0.072 \\
\hline$\leq 9$ & 55 & $44.15 \pm 4.03$ & $(0.453)$ & $(0.569)$ \\
\hline \multicolumn{5}{|c|}{ Health insurance coverage } \\
\hline Yes & 44 & $44.6 \pm 3.8$ & 1.864 & -0.246 \\
\hline No & 21 & $42.7 \pm 4.3$ & $(0.067)$ & $\left(0.048^{*}\right)$ \\
\hline \multicolumn{5}{|l|}{ Caregivers } \\
\hline \multicolumn{5}{|l|}{ Age (years) } \\
\hline$>50$ & 35 & $43.7 \pm 3.6$ & -0708 & 0.057 \\
\hline$\leq 50$ & 30 & $44.4 \pm 3.5$ & $(0.482)$ & $(0.652)$ \\
\hline \multicolumn{5}{|l|}{ Sex } \\
\hline Male & 30 & $43.7 \pm 4.5$ & 0.708 & 0.159 \\
\hline Female & 35 & $44.4 \pm 3.7$ & $(0.482)$ & $(0.206)$ \\
\hline \multicolumn{5}{|l|}{ Marital status } \\
\hline Regular partner & 55 & $44.1 \pm 3.9$ & 0.583 & -0.046 \\
\hline No regular partner & 10 & $43.3 \pm 4.7$ & $(0.562)$ & $(0.718)$ \\
\hline \multicolumn{5}{|l|}{ Occupation } \\
\hline Employed & 28 & $43.8 \pm 4.4$ & -0.407 & 0.086 \\
\hline Unemployed & 37 & $44.2 \pm 3.8$ & $(0.685)$ & $(0.494)$ \\
\hline \multicolumn{5}{|l|}{ Educational level (years) } \\
\hline$>9$ & 31 & $45.3 \pm 3.7$ & 2.616 & -0.316 \\
\hline$\leq 9$ & 34 & $42.8 \pm 4.0$ & $\left(0.011^{*}\right)$ & $\left(0.010^{*}\right)$ \\
\hline \multicolumn{5}{|l|}{ Relationship to patients } \\
\hline Parents & 5 & $40.2 \pm 5.8$ & 5.466 & 0.355 \\
\hline Spouse & 35 & $43.3 \pm 3.6$ & $\left(0.007^{*}\right)$ & $(0.004 *)$ \\
\hline Siblings or relatives & 25 & $45.6 \pm 3.6$ & & \\
\hline \multicolumn{5}{|c|}{ Self-perceived health status } \\
\hline Good & 4 & $43.5 \pm 4.8$ & 0.928 & -0.163 \\
\hline Moderate & 48 & $44.4 \pm 3.8$ & $(0.401)$ & $(0.194)$ \\
\hline Poor & 13 & $42.7 \pm 91.3$ & & \\
\hline \multicolumn{5}{|c|}{ Time of caring per day (hours) } \\
\hline$>8$ & 54 & $44.2 \pm 4.1$ & 0.975 & -0.124 \\
\hline$\leq 8$ & 11 & $42.9 \pm 3.5$ & $(0.333)$ & $(0.325)$ \\
\hline \multicolumn{5}{|l|}{ Living with patients } \\
\hline Yes & 48 & $43.08 \pm 3.97$ & -3.263 & 0.362 \\
\hline No & 17 & $46.53 \pm 2.98$ & $(0.002 *)$ & $(0.003 *)$ \\
\hline
\end{tabular}

were also summarized in Table 1. Of the four demographic factors associated with the patients, age and health insurance coverage were correlated with the level of caregiver burden. Caregivers of patients of older age (>50 year-old) and those who had no health insurance experienced more stress than the other demographic groups. With respect to the demographic factors associated with caregivers, only three following factors were found to be relevant; educational level, caregiver's relationship with the patient, and whether or not the caregiver lived with the patient. Caregivers with a high school education or higher (>9 years) were subject to more stress than those with a middle-school education or lower ( $\leq 9$ years). Caregiver's relationship to the patient also influenced his/her level of burden. Siblings or other relatives as caregivers had a higher level of burden than spouses $(p=0.023)$ or parents $(p=0.004)$. Besides, caregivers who did not live with the patients reported more stress than those who did. According to the stepwise regression analysis (Table 2 ), two most predictive 


\begin{tabular}{|c|c|c|c|c|c|c|}
\hline \multirow[t]{2}{*}{ Variable } & \multirow[t]{2}{*}{$\beta$} & \multirow[t]{2}{*}{ B } & \multicolumn{2}{|c|}{$95 \% \mathrm{CI}$ for $\mathrm{B}$} & \multirow[t]{2}{*}{$\mathrm{t}$} & \multirow[t]{2}{*}{$p$} \\
\hline & & & Low & High & & \\
\hline Constant & & 40.747 & 36.433 & 45.060 & 18.883 & $0.000^{*}$ \\
\hline Relationship to patients & 0.392 & 2.575 & 1.087 & 4.064 & 3.458 & $0.001^{*}$ \\
\hline Health insurance coverage & -0.240 & -2.045 & -3.973 & -0.117 & -2.120 & $0.038^{*}$ \\
\hline
\end{tabular}

factors for caregiver burden was patient's health insurance coverage and caregiver's relationship with the patient, respectively, in agreement with the correlation analysis.

\section{DISCUSSION}

To the best of our knowledge, the current study presented the first report of caregiver burden associated with RA patients with self-care deficit in China. We detected a moderate level of burden defined by $\mathrm{CBI}$ among the informal caregivers included in our study. Among five domains of $\mathrm{CBI}$, developmental burden had the highest scores. Furthermore, five items of developmental burden, all showing higher scores than items of other domains, indicated that caregivers of RA patients might suffer greatest stress associated with loss of control of their life. In our study, caregivers seemed to be mostly discouraged by disturbance of their normal life. The opportunity to develop their preferred way of life might be hindered due to long-term caring. In addition, three out of five items in the domain of time-dependent burden scored more than two points, suggesting the concern over the loss of control on time. Nursing strategy thus should be focused on enforcing rigid disease-control measures for RA patients, as well as strengthening the positive feeling of their caregivers. A stable disease condition may allow the caregivers to engage in activities of their own interest. In the meantime, developing a sense of fulfillment or achievement may help to lessen the impatience and fatigue caused by seemingly endless chores.

Beckham et al. ${ }^{6}$ first reported caregiver burden associated with RA patients in 1995. Using Zarit Burden Interview, they demonstrated that patients' condition and self-efficacy for controlling the joints were two main factors affecting caregiver burden. Furthermore, Riemsma et al. ${ }^{7}$ used a simplified CBI, in which the domain of emotional burden was omitted, to assess the caregiver burden of RA patients in the Netherlands. The mean CBI score of 174 patients in their study was 15.9, categorized as a mild level of caregiver burden. The domains with descending scores were as follows: time-dependent burden, developmental burden, physical burden, and social burden. The CBI mean score in our study was considerably higher, including the scores of four respective domains, than those of Riemsma et al. $^{7}$ The differences might be attributed to different study populations. In the study of Riemsma et al., ${ }^{7}$ the participants were patients simply fulfilling the diagnostic criteria proposed by American Rheumatism Association. ${ }^{8}$ By contrast, participants in our study were RA patients with self-care deficit due to joint damage. The latter tend to present with more severe conditions, thus called for better care in terms of time and quality, which would naturally aggravate caregivers' burden. On the other hand, this discrepancy may also be relevant with distinct healthcare maintenance systems in two countries. In China, chronic disease management program of RA has yet to be established. Repeated hospitalizations due to poor management of RA symptoms are not uncommon, which may also add to the stress of caregivers. In addition, emotional distress seems to be a growing concern for RA caregivers as disease progresses. In the report of Riemsma et al., ${ }^{7}$ the domain of emotional burden was omitted since the authors considered it insignificant. However, das Chagas Medeiros et al. $^{8}$ revealed that $59.6 \%$ of caregivers of severe RA patients suffered from emotional disturbance. Our study further supported this with the score of emotional burden being 6.95, encouraging professional consultation with psychologists. 
In their study, Jacobi et al. ${ }^{9}$ reported the caregiver burden of severe RA patients using Caregiver Reaction Assessment. Caregiver Reaction Assessment consists of five domains, four of which were designed to evaluate negative experience (lack of family support, financial problems, disrupted schedule, and loss of physical strength), and one for positive experience (carederived self-esteem). ${ }^{10}$ In their report, negative burden of RA caregivers were mostly caused by the domain of disrupted schedule, while least by the domain of loss of physical strength. These findings were further confirmed by Brouwer et al. ${ }^{11}$ Although it is difficult to compare the general burden level based on studies using two different assessment tools, it is interesting to note that the domain of disrupted schedule in Caregiver Reaction Assessment was essentially similar to that of developmental burden in $\mathrm{CBI}$. Both domains were shown to be most burdensome in all three studies, with loss of physical strength as the least burdensome one. Despite the vast differences between China and the Netherlands, a cross-cultural similarity may exist in terms of distribution of burden attributes for RA caregivers.

Among various demographic variables associated with RA patients, age and health insurance coverage affected the burden level significantly in this study conducted in the First Affiliated Hospital of Nanjing Medical University. Patients of older age aggravated the burden of their caregivers probably due to more advanced level of self-care deficit. For patients without health insurance in China, their informal caregivers, like spouses or siblings, are mostly likely to be financially involved which may cause more stress. On the other hand, of the factors associated with caregivers, relationship with the patients was found to be the most significant factor predictive for burden level. Unsurprisingly, educated and non-partner caregivers tended to have more expectations for their own life and might feel frustrated due to being confined to long-term caring for RA patients. Unexpectedly, age and sex of the caregiver had no influence on the burden level in our study. Previous studies showed that younger caregivers experienced greater burden than older ones ${ }^{12}$ and that females suffered more than males. ${ }^{12,13}$ Our results, however, was not in accordance with these findings. Most of the demographic factors investigated in our study are unlikely to change in the future except for the health insurance. Thus, purchasing and maintaining adequate health insurance coverage might aid in alleviating the caregiver burden of RA patients.

This study is mainly limited by its relatively small sample size. Future investigation should include a larger sample size across a wider range of cities in China to further validate our findings.

In conclusion, caregiver burden of RA patients with self-care deficit should be reduced as this burden might contribute to adverse health outcomes. ${ }^{14}$ Despite its small sample size, our study may assist in identifying the caregivers at risk of high level of burden and developing supportive strategies and interventions which target the patients and their caregivers to consolidate the sustainable care provided both at hospital and home.

\section{Declaration of conflicting interests}

The authors declared no conflicts of interest with respect to the authorship and/or publication of this article.

\section{Funding}

The authors received no financial support for the research and/or authorship of this article.

\section{REFERENCES}

1. Scott DL, Wolfe F, Huizinga TW. Rheumatoid arthritis. Lancet 2010;376:1094-108.

2. Sisk RJ. Caregiver burden and health promotion. Int J Nurs Stud 2000;37:37-43.

3. Aletaha D, Neogi T, Silman AJ, Funovits J, Felson DT, Bingham CO 3rd, et al. 2010 Rheumatoid arthritis classification criteria: an American College of Rheumatology/European League Against Rheumatism collaborative initiative. Arthritis Rheum 2010;62:2569-81.

4. Novak M, Guest C. Application of a multidimensional caregiver burden inventory. Gerontologist 1989;29:798-803.

5. Chou KR, Jiann-Chyun L, Chu H. The reliability and validity of the Chinese version of the caregiver burden inventory. Nurs Res 2002;51:324-31.

6. Beckham JC, Burker EJ, Rice JR, Talton SL. Patient predictors of caregiver burden, optimism, and pessimism in rheumatoid arthritis. Behav Med 1995;20:171-8. 
7. Riemsma RP, Taal E, Rasker JJ, Klein G, Bruyn GAW, Wouters JMG, et al. The burden of care for informal caregivers of patients with rheumatoid arthritis. Psychol Health 1999;14:773-94.

8. das Chagas Medeiros MM, Ferraz MB, Quaresma MR. The effect of rheumatoid arthritis on the quality of life of primary caregivers. J Rheumatol 2000;27:76-83.

9. Jacobi $\mathrm{CE}$, van den Berg $\mathrm{B}$, Boshuizen $\mathrm{HC}$, Rupp I, Dinant HJ, van den Bos GA. Dimensionspecific burden of caregiving among partners of rheumatoid arthritis patients. Rheumatology (Oxford) 2003;42:1226-33.

10. Given CW, Given B, Stommel M, Collins C, King S, Franklin $\mathrm{S}$. The caregiver reaction assessment (CRA) for caregivers to persons with chronic physical and mental impairments. Res Nurs Health 1992;15:271-83.
11. Brouwer WB, van Exel NJ, van de Berg B, Dinant HJ, Koopmanschap MA, van den Bos GA. Burden of caregiving: evidence of objective burden, subjective burden, and quality of life impacts on informal caregivers of patients with rheumatoid arthritis. Arthritis Rheum 2004;51:570-7.

12. Nijboer C, Triemstra M, Tempelaar R, Mulder M, Sanderman R, van den Bos GA. Patterns of caregiver experiences among partners of cancer patients. Gerontologist 2000;40:738-46.

13. Gallicchio L, Siddiqi N, Langenberg P, Baumgarten $\mathrm{M}$. Gender differences in burden and depression among informal caregivers of demented elders in the community. Int J Geriatr Psychiatry 2002;17:154-63.

14. Schulz R, Beach SR. Caregiving as a risk factor for mortality: the Caregiver Health Effects Study. JAMA 1999;282:2215-9. 